\title{
CORRELACIÓN ENTRE PH SALIVAL Y CARIES DENTAL EN PACIENTES CON SÍNDROME DE DOWN QUE ACUDEN A LA FUNDACIÓN ASISTENCIAL ARMADA NACIONAL-GUAYAQUIL-ECUADOR
}

\author{
CORRELATION BETWEEN SALIVARY PH AND DENTAL CARIES IN PATIENTS \\ WITH DOWN SYNDROME THAT COMES TO THE NATIONAL ARMED \\ ASSISTANCE FOUNDATION-GUAYAQUIL-ECUADOR \\ CORRELAÇÂOO ENTRE PH SALIVAL E CÁRIE DENTÁRIA EM PACIENTES COM \\ SÍNDROME DE DOWN QUE VÃO PARA A FUNDAÇ̃̃O NACIONAL DE \\ ASSISTÊNCIA ARMADA-GUAYAQUIL-EQUADOR
}

\author{
PAOLA CONTERO MEJÍA', MARÍA JOSÉ CABRERA DÁVILA' \\ ${ }^{1}$ Universidad Católica de Santiago de Guayaquil; Guayaquil, Ecuador
}

RESUMEN

Objetivo: determinar la correlación entre pH salival y caries dental en pacientes con Síndrome de Down que acuden a la Fundación Asistencial Armada Nacional (FASAN)-Guayaquil. Metodología: esta investigación se llevó a cabo en pacientes con Síndrome de Down por una muestra de 58 individuos entre las edades de 4 a 25 años de ambos géneros. Se realizó la medición del $p H$ salival a través de tiras indicadoras de pH colocadas sublingualmente, para generar diagnósticos de caries dental, índice de placa dental así como realizar encuestas de hábitos dietéticos a padres. Resultados: los pacientes con síndrome de Down, tuvieron un pH salival inicial neutro con un $50 \%$ pero sin gran diferencia al pH salival ácido con un $43 \%$, al contrario el pH salival que se tomó después de 10 minutos de la ingesta de bebidas azucaradas, prevaleció el pH ácido con un 83\% teniendo relación con la cantidad de caries encontradas en la muestra dentro de los rangos (bajo riesgo de caries $57 \%$ y moderado $22 \%$ ) y placa dental presente en un $59 \%$ con nivel 2 de placa dental. Se verificó más frecuente el riesgo de caries en el sexo masculino con $59 \%$ y el rango de edad más afectado fue de 12 a 18 años. Conclusión: existe una estrecha relación entre el pH salival ácido y la caries ya que se encontró en todos los pacientes con una prevalencia entre los rangos bajo y moderado, con un alto nivel de placa dental Nivel 2 de placa dental) debiéndose principalmente a la poca higiene bucal.

PALABRAS CLAVE: pH salival, caries dental, placa dental, bebidas azucaradas.

ABSTRACT

Objective: determine the correlation between salivary $\mathrm{pH}$ and dental caries in patients with Down Syndrome who attend the National Armed Assistance Foundation (NAAF) -Guayaquil. Methodology: this research was carried out in patients with Down Syndrome with a sample of 58 individuals between the ages of 4 to 25 years of both genders. Salivary $\mathrm{pH}$ measurement was carried out through pH indicator strips placed sublingually, with the purpose of generating diagnosis of dental caries, dental plaque index as well as to carry out dietary habit's surveys to parents. Results: the patients with Down syndrome had a neutral initial salivary $\mathrm{pH}$ of $50 \%$ but without much difference to the acidic salivary $\mathrm{pH}$ with $43 \%$, on the contrary, the salivary $\mathrm{pH}$ that was taken after 10 minutes of the intake of sugary drinks, the acidic $\mathrm{pH}$ prevailed with $83 \%$ being related to the number of cavities found in the sample within the ranges (low risk of caries $57 \%$ and moderate $22 \%$ ) and dental plaque present in $59 \%$ with level 2 dental plaque. The risk of caries was more frequently, verified in males with $59 \%$, and the most affected age range was 12 to 18 years. Conclusion: there is a close relationship between acidic salivary $\mathrm{pH}$ and caries since it was found in all patients with a prevalence between the low and moderate ranges, with a high level of dental plaque Level 2 dental plaque) mainly due to low oral hygiene.

KEYWORDS: salivary ph, dental caries, dental plaque, sugar sweetened beverages.

RESUMO

Objetivo: determinar a correlação entre pH salivar e cárie dentária em pacientes com Síndrome de Down que frequentam a Fundação Nacional de Assistência Armada (FASAN) -Guayaquil. Metodologia: esta pesquisa foi realizada em pacientes com Síndrome de Down por uma amostra de 58 indivíduos com idades entre 4 e 25 anos de ambos os sexos. A mensuração do pH salivar foi realizada por meio de tiras indicadoras de pH colocadas sublingualmente, para gerar diagnósticos de cárie dentária, índice de placa dentária e também para realizar pesquisas de hábitos alimentares aos pais. Resultados: os pacientes com síndrome de Down apresentaram pH salivar inicial neutro com 50\% mas sem grande diferença para o pH salivar ácido com 43\%, ao contrário do pH salivar que foi medido após 10 minutos da ingestão de bebidas açucaradas, as ácidas 0 pH prevaleceu com $83 \%$ sendo relacionado à quantidade de cáries encontradas na amostra dentro das faixas (baixo risco de cárie $57 \%$ e moderado 22\%) e placa dentária presente em $59 \%$ com placa dentária nível 2.0 risco de cárie foi verificado com maior frequência no sexo masculino com $59 \%$ e a faixa etária mais acometida foi de 12 a 18 anos. Conclusión: existe una estrecha relación entre el pH salival ácido y la caries ya que se encontró en todos los pacientes con una prevalencia entre los rangos bajo y moderado, con un alto nivel de placa dental Nivel 2 de placa dental) debiéndose principalmente a la poca higiene bucal PALAVRAS-CHAVE: ph salivar, cárie dentária, placa dentária, bebidas adocadas com acúcar. 
INTRODUCCIÓN

El síndrome de Down es una alteración genética que se presenta como consecuencia de una distribución anormal de cromosomas en donde existe un cromosoma extra en el par 21 conocido tam-

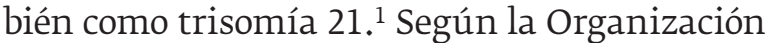
Mundial de la Salud (OMS) el 3\% de la población mundial tiene este tipo de enfermedad siendo África y Asia los continentes con mayor prevalencia y Latinoamérica ocupando el quinto lugar. ${ }^{2}$ El síndrome de Down se presenta en niños y se encuentra entre $1 / 700$ a 1/1000 nacidos vivos de la población general sin distinción de raza, nacionalidad, religión o de status-socioeconómico. ${ }^{1}$

Los pacientes con síndrome de Down presentan anomalías físicas y múltiples desórdenes cardíacos, inmunológicos, respiratorios, hematológicos, musculares y esqueléticos. Además presenta anomalías craneofaciales como un pobre desarrollo maxilofacial y anomalías dentarias como agenesias mal oclusión y retardo en la erupción..$^{2,3}$ Sus complicaciones médicas hacen que la salud oral sea de poco interés clínico, como la caries que es una de las enfermedades principales que afectan a estos individuos aparte de otras patologías orales y periodontales, ${ }^{2,3}$

Atienza et al (2012) han estudiado el flujo salival en los infantes con trisomía 21 llegando a la conclusión que esta es menor probablemente debido a cambios en la función secretora de las glándulas salivales; aunque en estos puede haber babeo que no estaría provocado por una hipersialia sino por tener la boca abierta, la posición adelantada de la lengua, y la hipotonía de la musculatura para deglutir. ${ }^{3}$

La dieta, la nutrición y los hábitos alimenticios constituyen el elemento más importante en la vida de los seres vivos, por lo que los factores $\mathrm{co}^{-}$ laterales son de gran importancia en el cuidado oral ya que pueden traer como consecuencia un cambio en $\mathrm{pH}$ salival y ayudar a la aparición de patologías como la caries dental.

Una nutrición con un alto consumo de azúcares refinados ayuda a la acidificación del $\mathrm{pH}$ bucal perjudicando el rol fundamental de remineralización que cumple la saliva para así evitar la acidificación de la misma y la caries dental. También se debe considerar otros factores que aumentan el potencial cariogénico como son la ingesta física de la dieta, el momento de la ingesta y la frecuencia. ${ }^{15,21}$
Según Alidianne, et al (2012) sostienen que estos sujetos presentan menor número de caries debido a un aumento de la salivación y al pH a los niveles anormales de bicarbonato y de estreptococos $\mathrm{mu}^{-}$ tans en el flujo salival. Además de morfología de fosas y fisuras menos pronunciadas, anodoncia, hipodoncia y una erupción retrasada de sus piezas dentales los que les haría menos susceptibles a las mismas. Mientras que sus índices CPO-D elevados se deben a los hábitos alimenticios y poca higiene bucal que es ofrecida por parte de sus familiares. ${ }^{6}$

Según Barrios, et al (2014) en estudios más recientes indica que la saliva de los pacientes Down presentan un elevado $\mathrm{pH}$, aumento en el contenido de sodio, calcio, ácido úrico, bicarbonato con una velocidad de secreción disminuida los cuales los hace más susceptibles a padecer caries y enfermedades periodontales. ${ }^{2}$

Este estudio tiene como objetivo determinar la relación entre el pH salival y el riesgo cariogénico dental antes y después de haber ingerido bebidas azucaradas en los pacientes con síndrome de Down que acuden a FASAN-Guayaquil.

\section{METODOLOGÍA}

Estudio descriptivo, transversal o prospectivo. La población del estudio la conformaron 64 niños, con síndrome de Down entre las edades de 4-25 años de ambos géneros, de la Fundación Asistencial Armada Nacional de la ciudad de Guayaquil- Ecuador en el año 2017 cumpliendo con los criterios de selección para la muestra, esta culminó con 58 participantes, debido a la no autorización de sus representantes para la participación en el presente estudio.

Los criterios de inclusión fueron:

- Pacientes con síndrome de Down que acudan regularmente a la fundación asistencial armada nacional (FASAN) de la ciudad de Guayaquil.

- Pacientes entre las edades de 4 a 25 años.

- Pacientes que estuvieran presentes durante el estudio.

Los criterios de exclusión fueron:

- Pacientes con poca colaboración.

- Pacientes que no tengan la autorización de sus padres.

- Pacientes que no logren entender las indicaciones. 
Antes de empezar a recoger la muestra se les entregó a los padres de familia un consentimiento informado donde otorguen la autorización para la participación de sus representados.

Además se les entregó una encuesta sobre los hábitos dietéticos de sus hijos para determinar los momentos y la cantidad de azúcar ingerida.

Se creó una ficha de historia clínica donde se incluyó contenidos personales, los índices de CPO-D y ceo-d de las caries, medición del pH salival y los índices de placa del paciente.

En la primera parte del estudio se realizó la medición del $\mathrm{pH}$ salival por medio de tiras indicadoras de las concentraciones ácidas o básicas que contiene la saliva. La muestra del $\mathrm{pH}$ salival se la recolectó antes y después de 10 minutos de haber ingerido bebidas azucaradas.

La toma de la primera muestra se realizó antes del receso donde los participantes se encuentran sin haber ingerido alimentos o bebidas dentro de dos horas tomando en cuenta que el $\mathrm{pH}$ salival se regulariza dentro de este tiempo, se les colocó una tira indicadora por debajo de la lengua por unos segundos, se retiró de la boca y se esperó hasta que esta tome el color de acuerdo al estado que se encuentre la saliva ya sea de acidez, neutralidad o alcalinidad. La segunda muestra de $\mathrm{pH}$ salival se la realizó 10 minutos después de haber hecho el receso donde los participantes consumieron bebidas azucaradas como parte de su dieta.

Se realizó un examen oral completo a cada uno de los pacientes donde se registró la sumatoria del número de caries, las piezas perdidas, obturadas y por extraer a través de los índices CPO-D y ceo-d tanto para dientes permanentes como temporales.

Luego se realizó la revisión del índice de placa dental con la aplicación de líquido revelador donde se registró todas las superficies teñidas en el diagrama presente en la historia clínica escogiendo la columna de las piezas más representativas que se encuentren en boca de cada paciente analizando los cuatro sitios principales del diente vestibular, palatino, mesialy distal.

Se analizaron las siguientes variables: síndrome de Down, sexo, edad, caries dental, placa dental, bebidas azucaradas, $\mathrm{pH}$ salival.
Posteriormente se elaboró un análisis estadístico descriptivo de frecuencia y porcentajes para las variables cualitativas, mientras que para las variables cuantitativas se realizó un promedio. Se tabuló la información utilizando un software estadístico R SPSS para el análisis de dichos datos y se usó Microsoft Office Execel ${ }^{\circledR} 2010$ (Microsoft Corporation, EEUU) en la sección de los gráficos.

Se ejecutó primero un análisis univariado a través de tablas de frecuencia y gráficos de barras a histogramas. Se realizó el análisis bivariado donde se utilizó para determinar asociación entre las variables numéricas, el test T student.

RESULTADOS

Posterior a la evaluación realizada a 58 pacientes con síndrome de Down se pueden determinar las siguientes características.

La figura 1 donde se valora el $\mathrm{pH}$ de los pacientes en un total de la muestra nos indica que en el pH inicial prevalece el neutro con un 50\% (29 pacientes) seguido del $\mathrm{pH}$ ácido con un $43 \%$ ( 25 pacientes) y solo un $7 \%$ (4 pacientes) del alcalino; posterior a la ingesta de bebidas azucaradas se encuentra una prevalencia de un $\mathrm{pH}$ ácido en un $83 \%$ (48 pacientes) seguido de un $\mathrm{pH}$ neutro de $12 \%$ (7 pacientes) y solo un $5 \%$ (3 pacientes) de alcalino.

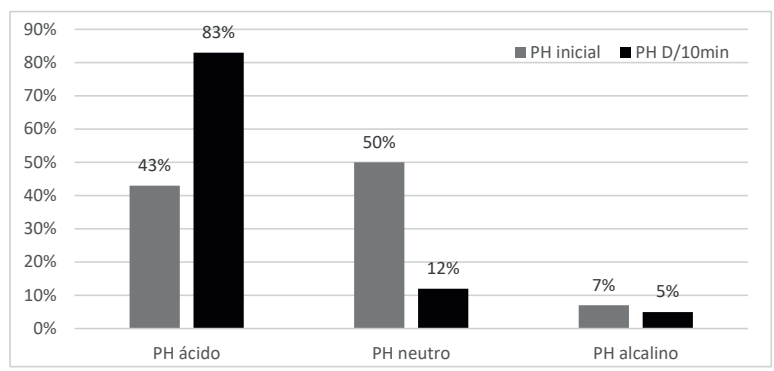

Figura 1. Prevalencia de pH salival inicial y pH salival después de 10 minutos del consumo de bebidas azucaradas.

En la figura 2, se registra los 58 pacientes por los rangos específicos de acuerdo al número de caries, en donde el rango con más prevalencia fue el bajo (7 a 12 caries) con el 57\% de la muestra. Además se relacionó el pH salival inicial y el pH salival posterior a la ingesta de bebidas azucaradas (10 minutos) en lo que se pudo observar, que entre los rangos muy bajo (17\%-10 pacientes) y el rango bajo de caries ( $57 \%-33$ pacientes) prevalece el $\mathrm{pH}$ neutro mientras que los rangos moderado $(22 \%-13$ pacientes) y alto ( $3 \%$ - 2 pacientes) prevalece el $\mathrm{pH}$ ácido, y posterior a la ingesta de bebidas azucaradas se verificó una prevalencia de $\mathrm{pH}$ salivalácido. 


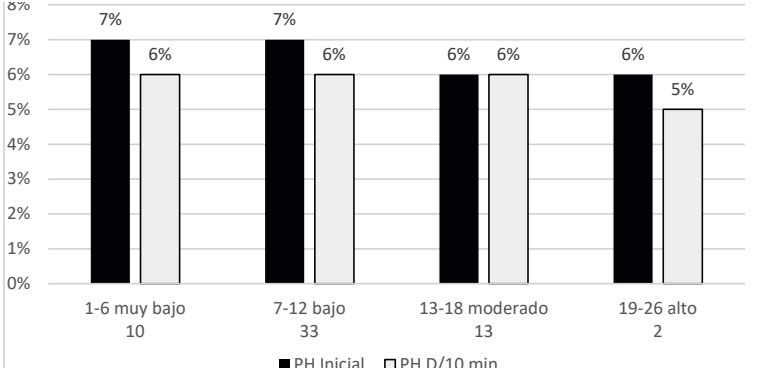

Figura 2. Prevalencia de rangos de riesgos de caries relacionados con el pH salival inicial y con el pH salival después de 10 minutos.

En la figura 3 de acuerdo a la muestra realizada $(10 \%)$, las 3 bebidas más consumidas por los encuestados, predominan los jugos empacados $24 \%$ el cual predomina un $\mathrm{pH}$ ácido del $23 \%$ y solo el $1 \%$ de $\mathrm{pH}$ alcalino; seguido del consumo de jugos naturales en un $22 \%$ obteniendo también un $\mathrm{pH}$ ácido en su totalidad y en tercer lugar el consumo de lácteos en un $19 \%$ donde predomina un $\mathrm{pH}$ ácido en un $18 \%$ y solo un $1 \%$ de $\mathrm{pH}$ alcalino. Esta información se obtuvo en la valoración del pH inicial y del pH después del consumo de bebidas azucaradas (10 minutos) en donde llamó la atención el $\mathrm{pH}$ alcalino ya que se obtuvo un $7 \%$ (4 pacientes) en el $\mathrm{pH}$ inicial y un $5 \%$ (3 pacientes) en el pH después de 10 minutos del consumo de bebidas azucaradas lo que representa el 1\% de los jugos empacados y los lácteos.

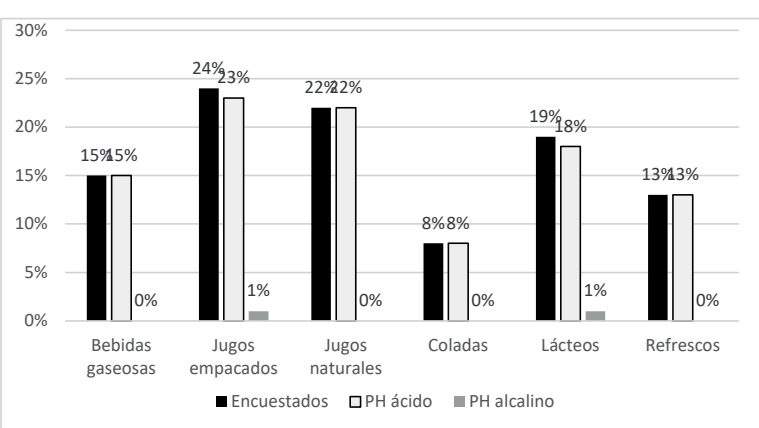

Figura 3. Encuesta de bebidas azucaradas más consumidas por los pacientes con síndrome de Down relacionados con el pH salival ácido y el pH salival alcalino.

En la figura 4 la muestra se dividió en tres grupos de edad: de 4 a 11 años (13 pacientes) prevalece el género masculino con un $12 \%$ (69 caries) y el género femenino en un $4 \%$ ( 25 caries), el grupo de 12 a 18 años (29 pacientes) prevalece el género masculino en un $24 \%$ (136 caries) seguido del género femenino en un $22 \%$ (128 caries), y el grupo de 19 a 25 años (16 pacientes) el género masculino tiene la mayor prevalencia de caries en un $24 \%$ ( 140 caries) y el género femenino un 14\% (82 caries); mientras que de acuerdo al género en una suma total de caries, el sexo masculino tiene la mayor prevalencia de caries con un 59\% (345 caries) ante un $41 \%$ ( 235 caries) del género femenino.

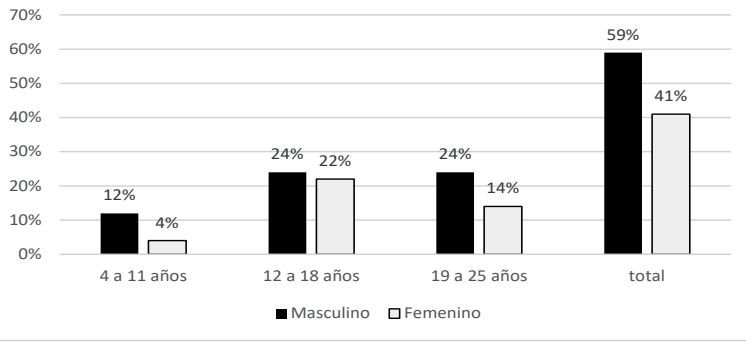

Figura 4. Prevalencia de caries de acuerdo a tres grupos de edad y al género.

En el nivel de placa dental se determina que el nivel 2 de placa, a nivel del tercio medio prevalece el 59\% que corresponde 34 de los 58 pacientes; seguido del nivel 1 de placa dental con un 56\% y con un nivel 3 de placa dental en un 16\%.

En la figura 5a se compara placa dental con el pH salival inicial a través de la cual se terminó que el $\mathrm{pH}$ neutro prevalece en los 3 niveles de placa dental, siendo sólo semejante al pH salival ácido con el $\mathrm{pH}$ salival neutro en un $47 \%$ (16 pacientes cada uno) en el nivel 2 de placa dental.

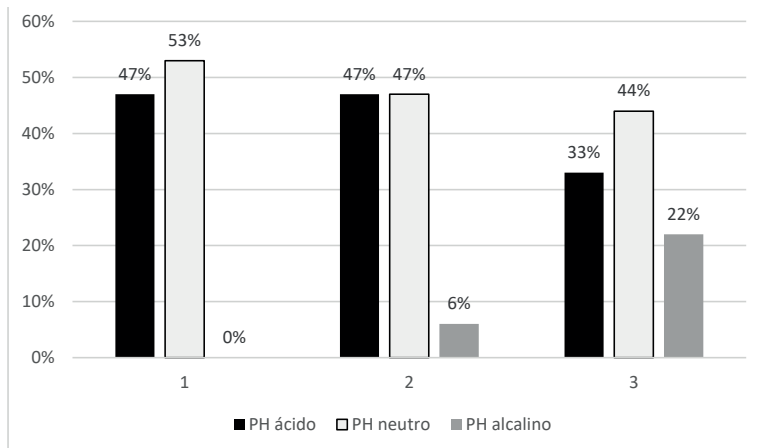

Figura 5a. Prevalencia de pH salival inicial en Los niveles de placa dental.

En la figura 5b se registra que después de la ingesta de bebidas azucaradas (10 minutos) el pH salival ácido prevalece en los 3 niveles de placa dental siendo más predominante en el nivel 3 de placa dental.

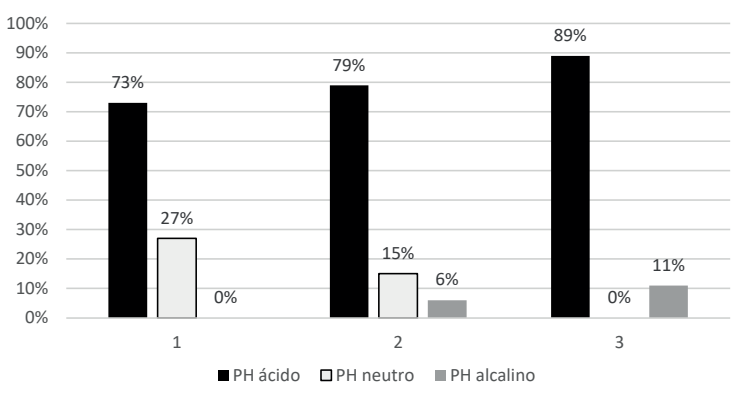

Figura $5 \mathbf{b}$. Prevalencia de pH salival después de 10 minutos de la ingesta de bebidas azucaradas en los niveles de placa dental.

De acuerdo a la encuestas realizadas a los representantes de los participantes de este estudio de acuerdo a la primera interrogante si ¿Cree Ud. que 
las bebidas azucaradas que consume su representado pueden causar daños en su salud bucal?, demostró que el $76 \%$ de los encuestados si sabía sobre los daños que pueden causar a la salud y el $24 \%$ revelo no saber del tema.

La siguiente pregunta encuestada iCon que frecuencia consume las bebidas azucaradas? se encontró que el $41 \%$ de los encuestados consume bebidas azucaradas 2 veces al día, seguida del 33\% que son consume 1 vez al día y un $26 \%$ que consume 3 veces al día. Estas bebidas son consumidas más en horarios de la mañana, seguida de los horarios de la tarde y por último en horarios de la noche. La edad desde que empiezan a consumirla se encuentra en un $46 \%$ en edades de 4 a 6 años, seguido del $43 \%$ en edades de 1 a 3 años y por ultimo un $9 \%$ en edades de 7 a 10 años.

DISCUSIÓN

La relación entre el $\mathrm{pH}$ salival y la caries dental en pacientes con síndrome de Down ha sido considerado un tema de controversia con pocos estudios sobre su estado bucal que indican que la caries dental es poco frecuente debido a diversas características encontradas en ellos como la composición de su saliva con un $\mathrm{pH}$ elevado, gran contenido de bicarbonato, ácido úrico o sus características orales como erupción retrasada, anomalías dentarias, fosas y fisuras poco profundas, agenesias, o por la cantidad de saliva que se debe a la posición adelantada de la lengua. Pero esto dependerá mucho de su manera de vida, de su alimentación y del cuidado dental que le sea proporcionado ya que ahí se puede formar una controversia si la caries dental es poco frecuente o no.

El estado bucal de los pacientes con síndrome de Down se caracteriza por tener poca higiene oral con una retención prolongada de sus alimentos además se puede mencionar que los factores que lleven a un alto riesgo cariogénico tienen mucho que ver con la dieta del mismo, sólida como líquida además un mal cuidado en su higiene oral dada su discapacidad física, mental y por parte de sus responsables.

De acuerdo con lo que refiere Guerrero (2015) la gran mayoría de estudios relacionados sobre caries en infantes con síndrome de Down coinciden en afirmar que son pocos frecuentes.

Según los resultados obtenidos en este estudio difieren con dicho autor ya que se demuestra como la mala higiene oral y el consumo de bebidas azu- caradas puede producir cambios en el pH salival y llevar a una desmineralización del esmalte, produciendo la caries dental.

El análisis de este estudio demostró que existe un alto riesgo cariogénico que se logró corroborar gracias a la medición del $\mathrm{pH}$ salival, al diagnóstico de caries y la placa dental presentes en boca además de sus hábitos alimenticios y de su higiene oral. Se puede destacar que se encontró una variación en el $\mathrm{pH}$ salival donde en el inicial, el pH salival ácido se encuentra en un $43 \%$ y el pH salival neutro en un $50 \%$ ambos no tienen una diferencia significativa, mientras que en el $\mathrm{pH}$ salival después de 10 minutos prevalece el ácido $83 \%$ que coincide con los estudios de Barrios (2014). ${ }^{2}$

Además de la alta presencia de caries encontradas con una prevalencia en el sexo masculino concordando con Alidianne (2012)6 y Barrios (2014), al contrario en relación a las edades donde dicho investigador encontró más prevalencia de caries de 2 a 10 años, ${ }^{6}$ mientras que en este estudio la mayor prevalencia fue de 12 a 18 años.

El estudio coincide en cierta parte con los resultados de Alidianne (2012) y Guerrero (2015) que sostienen que estos pacientes pueden presentar sus índices CPO-D y ceo-d (caries) elevados debido a los hábitos alimenticios y poca higiene bucal que es ofrecida por parte de sus familiares, ya que en el estudio se demostró que todos los participantes poseían caries con más prevalencia en los rangos de bajo riesgo 57 ( 7-12caries) y moderado 22\% (1318 caries) de caries como también un alto índice de placa dental en un 59\% con el nivel 2 de placa dental.

En este estudio fue posible determinar la importancia de un correcto diagnóstico de todas las manifestaciones orales en estos paciente con síndrome de Down posibilitando que los cuidadores tengan más información sobre el control dental para así disminuir los riesgos orales y darles una mejor calidad de vida.

CONCLUSIÓN

El pH salival encontrado en los pacientes con síndrome de Down dio que en el pH salival inicial, un $\mathrm{pH}$ salival neutro sin mucha diferencia del $\mathrm{pH}$ salival ácido y un $\mathrm{pH}$ salival ácido predominando después de 10 minutos de la ingesta de bebidas azucaradas. Los pacientes con síndrome de Down entre las edades de 12 a 18 años presentan una mayor prevalencia de caries; y de la suma total de 
caries el sexo masculino predomina en la misma. Además de un alto índice de placa dental donde prevalece un $59 \%$ del nivel 2 de placa dental en la población. En virtud a los datos encontrados se puede afirmar que el consumo de bebidas azucaradas, la cantidad de placa dental y la falta de higiene oral crean un ambiente constantemente ácido provocando la desmineralización del esmalte y creando así una alta relación con la presencia de la caries dental.

\section{REFERENCIAS BIBLIOGRÁFICAS}

1. Bancalari, S.C \& oliva, M.P. Riesgo biológico de caries en niños con síndrome de Down entre 12 y 17 años del Cavime, Concepción, año 2010. Int. J. Odontostomat. 6(2): 221-224. 2012

2. Barrios C.E, Vila V.G, Martínez S.E, Encina A.J, Relación entre $\mathrm{pH}$ salival y caries dental en pacientes con síndrome de Down. Rev. Odontoestomatologia. Vol. XVINo. 23. Mayo 2014

3. Areias C., Pereira ML., Pérez D., Macho V., Coelho A., Andrade D. Enfoque clínico de niños con síndrome de Down en el consultorio dental. Av. Odontoestomatologia. 30(6):307-313. 2014.,

4. Bexi Perdomo., Deyla Torres., Yoly Paredes., SíndromedeDowneHigiene bucal: lineamientos para padres, cuidadores y docentes. RevVenezInvestOdont. IADR. 2(2): 156-169. 2014

5. Katiuska Rodríguez Guerrero, Rafael Claveria Clarck., Maritza Peña Sisto. Algunascaracterísticas clínico-epidemiológicas del Síndrome de Down y su repercusión en la cavidad bucal. MEDISAN 19(10): 1273. 2015

6. Alidianne Cabral X., Gordon-Núñez M., Ribeiro C., Calvacanti A., Lins R. Prevalencia de anormalidades bucales y caries dental en portadoresdeSíndromedeDown. Acta Odontológica Venezolana. Volumen $50 \mathrm{~N}^{\circ} 4.2012$

7. Ramos J., Galindo F., Torres C., Avilés. S. Síndrome de Down-Caso clínico. Odontología Actual. Año 5, núm., 57, enero del 2008.

8. Culebras Atienza E., Silvestre-Rangil J., Silvestre Donat F. Alteraciones Odonto-esmatologicas en el niño con síndrome de Down. Revista Española de Odontopediatria. 2012; 68(6): 434-436.

9. Díaz-CárdenasS., González-MartínezF. Prevalencia de caries dental y factores familiares en niños escolares de Cartagena de Indias, Colombia. Revista de salud pública 12(5): 843-851, 2010.

10. Lizama M., Retamales N., Mellado C. Recomendaciones de cuidados en salud de personas con síndrome de Down: 0 a 18 años. Revista Médica de Chile 141:80-89. 2013.

11. Gorduño-Zarazua L., Giammateo Alois L., Kofman-Epstein S., Cervantes Peredo A.
Prevalencia de mosaicismo para la trisomía 21 y análisis de las variantes citogenéticas en pacientes con diagnostico síndrome de Down. Revisión de 24 años (1986-2010) del servicio de genética del Hospital General de México “Dr. Eduardo Liceaga”. Bol. Med. Hosp.Infant.Mex. 70(1):31-36. 2013

12. Pérez Chávez D. Síndrome de Down. Revista de Actualización Clínica. V 45: 2357-2361. 2014.

13. Tirado Amador L., Díaz Cárdenas S., Ramos MartínezK. Salud bucalen escolares con síndrome de Down en Cartagena (Colombia). Revista Clínica Médica Familiar. 8(2):110-118. 2015.

14. Alonso Noriega M., Karakowsky L. Caries de la infancia temprana. Perinatol Reprod Hum.23 (2):90-97. 2009.

15. Criollo Chicaiza L. Valoración del pH salival asociadoal consumo de (Lácteos, yogurt natural y yogurt de durazno), en individuos con síndrome de Down de la fundación "El Triángulo" y su posible relación con la caries dental. Quito. Universidad Central del Ecuador. Marzo. 2015.

16. Cosio Arévalo D., Ortega Cambranis A., Vaillard Jiménez E. Determinación del pH salival antes, durante y después del consumo de caramelos en niños de 3,4 y 5 años de edad. Oral. Año 11. Núm. 35: 642-645. 2010.

17. Caridad C. ElpH, flujo salival y Capacidad Buffer en Relación a la formación de la Placa Dental. Odous Científica. Vol.IX. No. 1, Enero-Junio. 2008.

18. Franco G., SaabR., VarianiL., Torres M., Fragoneze A., BrancherJ. Analysis of salivary ph., flow rate, buffering capacity, concentrations of calcium, urea and total proteins in 2-8 years-old children whit Downs syndrome. RSBO. Jan-Mar, 11(1): 66-70. 2014.

19. Aguirre Aguilar A., Narro Sebastián F. Perfil salival y su relación con el índice CEOD en niños de 5 años. Revista Odontológica Mexicana. Vol.20 No. 3 Julio-Septiembre. 2016.

20. Pérez A. La Biopelicula: una nueva visión de la placa dental. Rev. Estomatológica Herediana. 15(1):82-85. 2005

21. Verdozoto J. Relación entre ingesta de caramelos ácidos, pH salival, estrato socioeconómico y erosión dental en niños de tercero a séptimo año de educación básica de la parroquia de Guayllabamba. Odontología. V.16 Diciembre 2014.

22. Aguirre A., Vargas A. Variación del pH salival por consumo de chocolate y su relación con el IHO en adolescentes. Oral. Año 13. Núm. 41: 857-861. 2012.

23. Molina J. Atención y cuidados odontológicos para los niños con síndrome de Down. Revista Síndrome de Down. 22:15-19, 2005. 
24. Barrios C., Martínez S., Encina A. Relación de los niveles de caries y $\mathrm{pH}$ salival en pacientes adolescentes. RAAO. Vol.LV. Num.1-2016.

25. Demicheri A., Batlle A. La enfermedad periodontal asociada al paciente con Síndrome de Down. Odontoestomatologia. Vol. XIII. No. 18. Noviembre 2011.

26. Soham B., Srilatha KT. Seema Salivary Characteristics of Downs Syndrome Children-A Review. Journal of Oral Hygiene \& Health. Vol.4:201. 2016.

27. Dávila M., Gil M., Daza D., Bullones X., Ugel E. Caries dental en personas con Retraso Mental y Síndrome de Down. Rev. Salud Pública. 8(3): 207-213. 2006.
28. Benítez M., López P., Yamamoto A. Enfermedad Periodontal en pacientes adolescentes con Síndrome de Down. Presentación de caso. Revista Odontológica Mexicana. Vol.18 Núm. 3. Julio-Septiembre. 2014

29. Morales C.M.C., Naukart G.Z.C. Prevalencia de maloclusiones en pacientes con síndrome de Down. Oral Año 10. Num.32: 537-539. 2009.

30. Capreli M. Síndrome de Down, hipotiroidismo y otras causas. El niño campesino deshabilitado. Retraso Mental. Capítulo 32: 277-282. 2013. 